\title{
Liver Bioengineering Using Decellularized Whole-Liver Scaffolds
}

\author{
Iris Pla-Palacín, Pilar Sainz-Arnal, Sara Morini, Manuel Almeida, \\ and Pedro M. Baptista
}

\begin{abstract}
Currently, due to the progress made in the field of regenerative medicine, whole-organ bioengineering is becoming a valid alternative to cope with the shortages of organs for transplantation. In this chapter, we describe the main techniques carried out for pig liver bioengineering, which serves as an essential model for future human liver bioengineering. These include porcine whole-liver decellularization, endothelial and mesenchymal stem cell isolation, porcine ES-derived hepatoblasts, and scaffold recellularization using a bioreactor perfusion system.
\end{abstract}

Keywords Decellularization, Liver scaffold, Organ bioengineering, Perfusion bioreactor system, Recellularization

\section{Introduction}

Due to its role in the production of several proteins, vitamins, lipids, and carbohydrates, the synthesis of substances necessary for homeostasis and digestion, detoxification of various metabolites, and glucose level regulation, the liver is one of the body's vital organs in metabolism and homeostasis.

The high mortality of end-stage liver disease is a global public health issue, to which liver transplantation is the only definitive treatment to improve the survival and quality of life of these patients. However, there is still a significant gap between organ supply and demand.

Whole-organ bioengineering and regenerative medicine are promising new technologies that can help reduce liver shortage by increasing the number of organs available for transplantation. In this perspective, decellularization is an attractive technique in regenerative medicine to prepare scaffolds. This consist of the removal of cells from a tissue or organ using detergent perfusion through the vasculature, leaving behind an intact structure of the extracellular material, which is fundamental to regulate cell differentiation and function. The perfusion decellularization method represents a useful 
Iris Pla-Palacín et al.

procedure to create whole-organ scaffolds ready to be recellularized with freshly isolated cells and maintained in bioreactors for cell expansion, differentiation, and function $[1,2]$. In this protocol, we describe how to prepare porcine liver scaffolds and isolate and expand endothelial, mesenchymal stem cells and iPS-derived hepatoblasts for posterior recellularization and maintenance of this bioengineered liver in a bioreactor perfusion system.

\section{Materials}

\subsection{Liver Harvesting and Cannulation}

\subsection{Decellularization and Scaffold Preparation for Bioreactor}

1. Scalpel.

2. Forceps (with and without teeth).

3. Scissors.

4. Silk suture 4-0.

5. Cannula 18G.

6. Straight fitting $6.4 \mathrm{~mm}$ diameter.

1. Distilled water.

2. Peristaltic pump with $14 \mathrm{G}$ and $17 \mathrm{G}$ tubing (Masterflex $\mathrm{L} / \mathrm{S}$ with Masterflex L/S easy load pump head, Cole Parmer).

3. Silicone tubing (silicone tubing size $14 \mathrm{G}$ and $17 \mathrm{G}$, Cole Parmer).

4. Pulse dampener.

5. Pressure sensor (APT Pressure Transduced, Panlab).

6. Pressure controller (Panlab).

7. Male Luer.

8. Four-way stopcock.

9. Detergent solution: $1 \%$ Triton X-100 with $0.1 \%$ ammonium hydroxide in distilled water.

10. X-ray source.

11. $150 \mathrm{~cm}$ culture dish.

\subsection{Cell Isolation and} Culture
1. Piglet umbilical cord.

2. PBS - (without calcium and magnesium).

3. PBS+ (with calcium and magnesium).

4. $0.05 \%$ trypsin/EDTA.

5. Neutralization medium: DMEM/F12 with $10 \%$ FBS.

6. $18 \mathrm{G}$ cannula.

7. Endothelial growth medium 2 .

8. Fibronectin $\left(5 \mu \mathrm{g} / \mathrm{cm}^{2}\right)$. 
9. 6-multiwell cell culture plate.

10. $150 \mathrm{~cm}$ cell culture dish.

11. $50 \mathrm{~mL}$ conical centrifuge tubes.

12. Pig femur.

13. Surgical saw.

14. $18 \mathrm{G}$ needle.

15. Scalpel.

16. Scissors.

17. Forceps.

18. Bovine gelatin $(0.02 \%)$.

19. 100 and $40 \mu \mathrm{m}$ cell strainers.

20. Histopaque-1077.

21. Centrifuge.

22. Incubator at $37{ }^{\circ} \mathrm{C}$ and $5 \% \mathrm{CO}_{2}$.

23. MEF medium: $81 \%$ DMEM high glucose with $15 \%$ FBS, $2 \%$ $\mathrm{P} / \mathrm{S}, 1 \% \mathrm{~L}$-Glut, 1\% DMEM nonessential amino acids.

24. Matrigel (Geltrex hESC-qualified reduced growth factor basement membrane) - diluted to $2 \mathrm{mg} / \mathrm{mL}$ in DMEM/F12 medium.

25. TrypLE.

26. 293FT cells.

27. Gelatin.

28. $100 \mathrm{~mm}$ tissue culture dish pre-coated with $0.1 \%$ gelatin.

29. DMEM high glucose.

30. 293FT medium: DMEM high glucose with 10\% FBS.

31. FuGENE HD Transfection Reagent.

32. rtTA plasmid.

33. STEMCCA (OKSM) Lentivirus Reprogramming Kit.

34. D8.9/psPAX2 plasmid.

35. VSV-G plasmid.

36. Microcentrifuge.

37. Sterile microcentrifuge tubes $(1.5 \mathrm{~mL})$.

38. $0.45 \mu \mathrm{m}$ filters.

39. Beckman Coulter Optima L-90K ultracentrifuge with SW-32 rotor.

40. $0.25 \%$ Trypsin- $0.53 \mathrm{mM}$ EDTA. 
Iris Pla-Palacín et al.

41. Polybrene.

42. Doxycycline.

43. Hemocytometer.

44. Pluripotent stem cell medium: mTeSRI.

45. RPMI cell differentiation medium: RPMI 1640, HEPES medium with $1 \%$ nonessential amino acids, $1 \% \mathrm{P} / \mathrm{S}$.

46. B27 without insulin.

47. B27 with insulin.

48. Activin A.

49. Bone morphogenetic protein 4.

50. Fibroblast growth factor 2 .

51. Hepatocyte growth factor.

52. EGM2 bullet kit (Lonza).

53. MCDB 131 culture medium (ThermoFisher Scientific).

54. DMEM/F12 culture medium (ThermoFisher Scientific).

2.4 Bioreactor System
1. Bioreactor vessel (Glass Ball Spinner, $250 \mathrm{~mL}$, Bellco Biotechnology Inc., Vineland, NJ, USA).

2. Peristaltic pump (same description as in Sect. 2.2, item 2).

3. Silicone tubing (same description as in Sect. 2.2, item 3).

4. Pressure sensor (same description as in Sect. 2.2, item 5).

5. Pressure controller.

6. Magneto.

7. Magnetic stirrer.

8. Pulse dampeners (Cole Palmer).

9. Four-way stopcocks (Cole Palmer).

10. Smart site connections (Cole Parmer).

11. Luer-lock syringe $20 \mathrm{~mL}$.

12. $20 \mu \mathrm{m}$ filter.

13. Empty (sterile and clean) culture media bottle (as a secondary reservoir).

14. Bioreactor culture medium: 50\% DMEM/F12 + 50\% MCDB 131 containing $5 \% \mathrm{FBS}, 1 \% \mathrm{~L}$-glutamine, $1 \%$ penicillin/streptomycin, $5 \mu \mathrm{g} / \mathrm{mL}$ insulin, $10 \mu \mathrm{g} / \mathrm{mL}$ transferrin, $50 \mu \mathrm{g} / \mathrm{mL}$ VEGF, $40 \mu \mathrm{g} / \mathrm{mL}$ EGF, $40 \mu \mathrm{g} / \mathrm{mL}$ FGF-2, and $40 \mu \mathrm{g} / \mathrm{mL}$ IGF-1. 
3 Methods

3.1 Pig Cadaveric Liver Harvesting

3.2 Liver Decellularization
1. The suprahepatic vena cava is dissected as close to the atrium as possible.

2. The common bile duct is dissected as close to the duodenum as possible.

3. The portal vein is carefully dissected from the surrounding tissue, to visualize it and its branches. Lateral branches are ligated with 4-0 silk suture and cut as close to the intestines as possible.

4. The hepatic artery is carefully dissected from the surrounding tissue, to visualize it and its branches. Lateral branches are ligated with silk suture 4-0 and cut as close to the stomach as possible.

5. Infra-hepatic vena cava is carefully dissected and cut without damaging the lobe. The cut should be performed before the renal bifurcation (see Note 1 ).

6. Liver attachments are cut to remove the intact organ.

7. The diaphragm is carefully dissected around the esophagus.

8. The portal vein is cannulated with a straight fitting of $6,4 \mathrm{~mm}$ diameter. The hepatic artery should be cannulated with a $20 \mathrm{G}$ cannula.

9. The gallbladder is emptied and cleaned.

Decellularization is usually performed at room temperature, after one freezing/thawing cycle. Scaffolds are kept sterile at $4{ }^{\circ} \mathrm{C}$ until use. Components are assembled as shown in Fig. 1, and results are seen in Fig. 2.

1. Cannulas are attached to a peristaltic pump by using $17 \mathrm{G}$ tubing for the portal vein and $14 \mathrm{G}$ for the hepatic artery.

2. $2 \mathrm{~L}$ of distilled water is perfused through the portal vein at a pressure of $25 \mathrm{mmHg}$ for both the hepatic artery and the portal vein.

3. $10 \mathrm{~L}$ of decellularization solution is perfused afterward (see Note 2).

4. $20 \mathrm{~L}$ of distilled water is perfused to remove the detergent from the tissue.

1. Umbilical cords are obtained from piglets that had undergone spontaneous abortions in farms of animal production.

2. Locate umbilical cord vein and inject $20 \mathrm{~mL}$ of PBS with a $18 \mathrm{G}$ cannula. 
Iris Pla-Palacín et al.

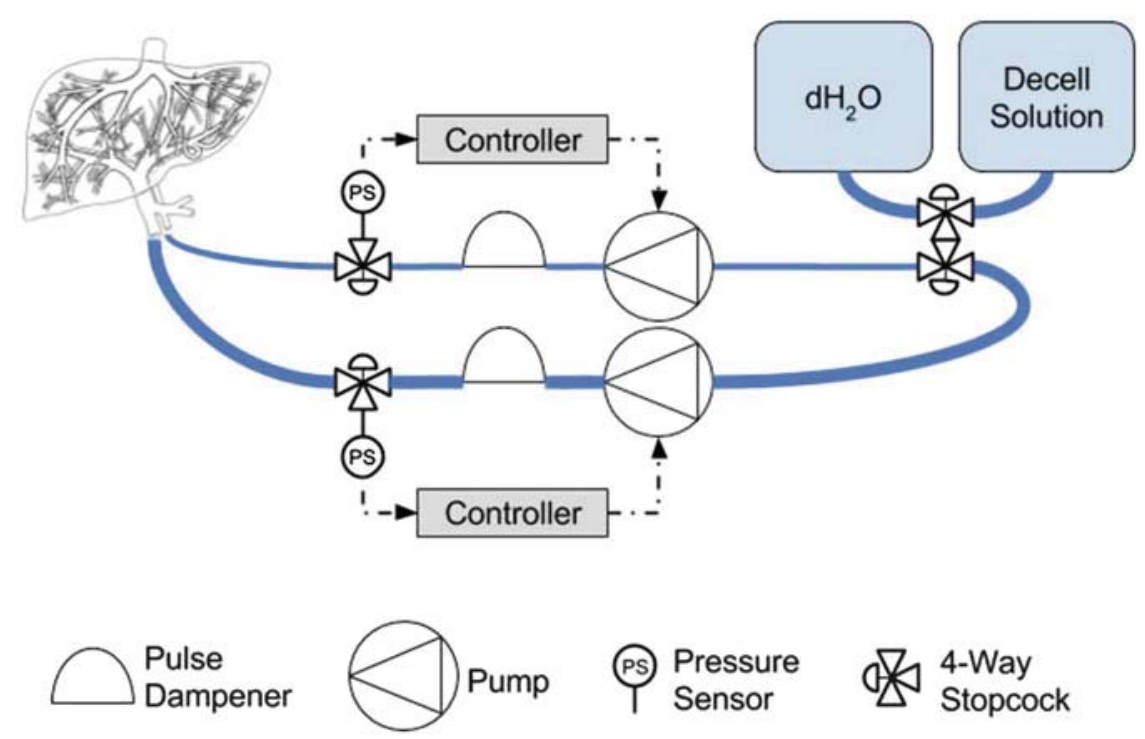

Fig. 1 Schematic diagram of the decellularization setup. The fine line represents the hepatic artery line; the thicker one represents the portal vein line. The liver is first washed with distilled water and then perfused with the decellularization solution detergent. Finally it is washed again to remove the detergent from the liver
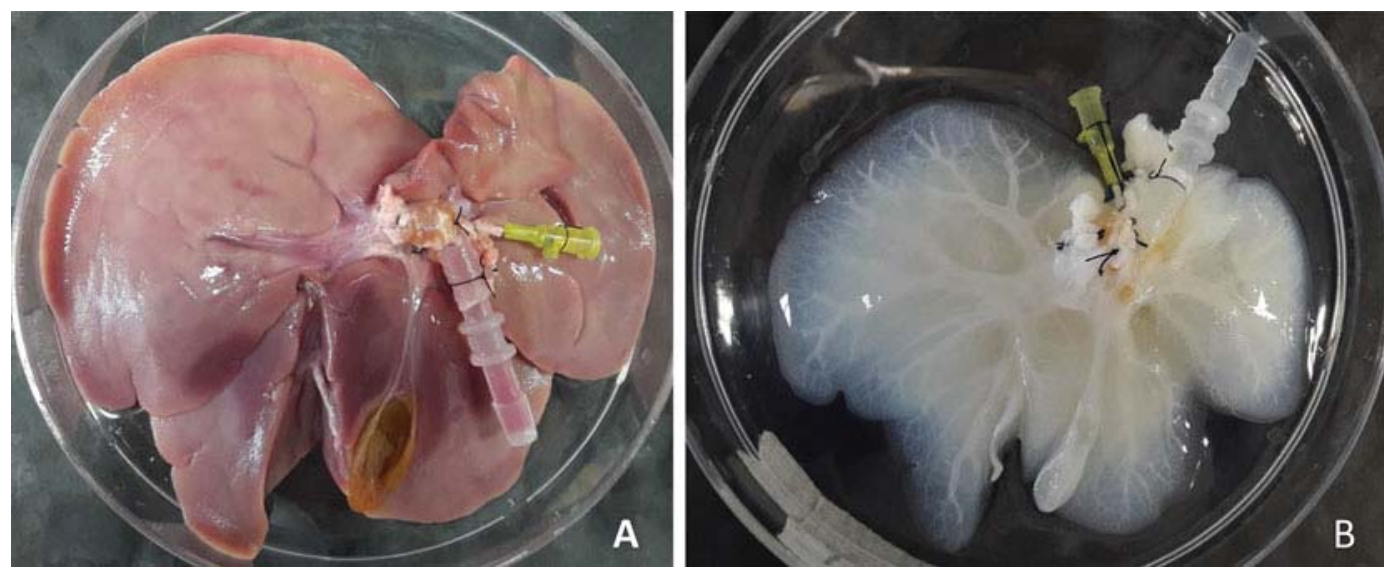

Fig. 2 Piglet liver before (a) and after decellularization (b)

3. Inject $20 \mathrm{~mL}$ of pre-warmed trypsin. Collect and inject again. Repeat this step for $7 \mathrm{~min}$.

4. Collect all the trypsin and neutralize with $20 \mathrm{~mL}$ of DMEM with $10 \%$ FBS and centrifuge $400 \times g$ for 5 min.

5. Aspirate the supernatant and resuspend the pellet in $10 \mathrm{~mL}$ of EGM-2 with $20 \%$ FBS.

6. Transfer the cells to a 6-multiwell dish coated with fibronectin $(0.03 \mathrm{mg} / \mathrm{mL})$, and place it into the incubator at $37^{\circ} \mathrm{C}$.

7. Allow the cells to grow for 3-4 days. Change media at day 4 . 
8. Once some colonies are detected, change media to EGM-2 with $10 \%$ FBS. Change media every 3 days.

9. Trypsinize the culture when it reaches $80 \%$ confluence (see Note $3)$.

3.4 pMSC Isolation

1. Pig bone marrows are isolated from cadaveric piglets slaughtered by farms of animal production due to malformations or trauma.

2. Femurs are carefully dissected and cleaned from muscle/ligament tissue.

3. Cut the epiphysis and flush the bone marrow with a $18 \mathrm{G}$ needle with $5 \mathrm{~mL}$ of DMEM and $1 \% \mathrm{P} / \mathrm{S}$.

4. Collect this cell suspension and centrifuge at $300 \times g$ for $5 \mathrm{~min}$.

5. Reconstitute the pellet with $10 \mathrm{~mL}$ of DMEM $+1 \% \mathrm{P} / \mathrm{S}$, and filter sequentially through 100 and $40 \mu \mathrm{m}$ cell strainers.

6. Separate with a histopaque density gradient centrifugation.

7. Resuspend the pellet with $10 \mathrm{~mL}$ of DMEM/F12 with $10 \%$ FBS and $1 \% \mathrm{P} / \mathrm{S}$, and plate the cells in a $10 \mathrm{~cm}$ culture dish previously coated with a bovine gelatin solution $(0.2 \%)$. Place it in the incubator.

8. Allow the cells to grow for 2 days. Change media at day 2 .

9. Trypsinize the culture when it reaches $70-80 \%$ confluence.

3.5 Generation of iPS-Derived Hepatoblasts

3.5.1 Lentiviral Production
Cultured porcine mesenchymal stem cells with less than four passages were reprogrammed to generate iPS cells by transduction with four human reprogramming factors: Sox2, Klf4, Oct4, and c-Myc. We followed the protocol published by Rajarajan et al [3]. Once the iPS cells were obtained and characterized properly, they were differentiated to hepatoblast-like cells.

1. Culture 293FT cells onto gelatinized $100 \mathrm{~mm}$ dish in 293FT media until cells reach $80-90 \%$ confluency.

2. Mix in microcentrifuge tube $770 \mu \mathrm{L}$ DMEM high glucose with $50 \mu \mathrm{L}$ FuGENE Reagent per $10 \mathrm{~cm}$ dish, and incubate for $5 \mathrm{~min}$ at room temperature.

3. Add $5.5 \mu \mathrm{L}$ VSV-G and $8.25 \mu \mathrm{L} \mathrm{D8.9}$ and mix well.

4. Add a total of $11 \mu \mathrm{g}$ vector DNA (STEMCCA Lentivirus Reprogramming Vector pluripotent transcription factors at $11 \mu \mathrm{g}$ /infection). Mix gently and incubate for $30 \mathrm{~min}$ at room temperature.

5. During incubation, add $10 \mathrm{~mL}$ of fresh $293 \mathrm{FT}$ medium to 293FT cells.

6. Add entire FuGENE/DNA complex to the $100 \mathrm{~mm}$ dish drop by drop, and rotate the plate to mix the contents. Incubate for 12-24 h. 
Iris Pla-Palacín et al.

7. Change 293FT medium 12-24 h later.

8. After $24 \mathrm{~h}$, collect all media and store at $4{ }^{\circ} \mathrm{C}$. Add $10 \mathrm{~mL}$ of fresh $293 \mathrm{FT}$ medium to the dish. Repeat every subsequent $24 \mathrm{~h}$ for three collections.

9. Filter collected viral medium through a $0.45 \mu \mathrm{m}$ filter, and centrifuge at $50,000 \times \mathrm{g}$ for $1.5 \mathrm{~h}$ at $4{ }^{\circ} \mathrm{C}$.

10. Decant supernatant and add $200 \mu \mathrm{L}$ of serum-free DMEM medium to the pellet and let it stand overnight at $4{ }^{\circ} \mathrm{C}$.

11. Resuspend, aliquot, and store the virus at $-80^{\circ} \mathrm{C}$.

3.5.2 Infection of Fibroblasts Using Pluripotency Transcription Factors
3.5.3 Hepatoblast-Like Cell Production
1. Culture pig MSC in a gelatin-coated single well of a 6-well plate until $90 \%$ confluency.

2. Trypsinize the cells in culture, count the number of cells, and adjust the cell concentration to $10^{4}$ cells $/ \mathrm{mL}$.

3. Add polybrene at a final concentration of $8 \mu \mathrm{g} / \mathrm{mL}$ to the cellcontaining medium. Mix by pipetting up and down.

4. Thaw the four viral constructs Oct-3/4, Sox 2, Klf4, and c-Myc, and combine them into a cocktail. Add into the cell mixture. Mix gently pipetting up and down (see Note 4 ).

5. Plate $1 \mathrm{~mL}$ of the cell/virus mixture onto a single well of a gelatin-coated 6-well plate, and incubate for $24 \mathrm{~h}$.

6. After this time, wash with warm MEF medium, replenish with $2 \mathrm{~mL}$ of fresh MEF medium, and continue incubation.

7. Leave the culture until iPS colonies appear. Once they do (around day 7), pick colonies with a $200 \mu \mathrm{L}$ pipet.

After post-viral transduction, the cells are grown on X-ray radiation inactivated mouse embryonic fibroblast (MEF) feeder layer with mTeSRl. After that, cells were adapted to grow onto Matrigel.

Differentiation of iPS cells into hepatoblast-like cells is induced as previously described [4].

1. Pig pluripotent stem cells should be maintained on Matrigelcoated $100 \mathrm{~mm}$ tissue culture dishes using mTeSRl (see Note $5)$.

2. After $50 \%$ of confluence, wash the cells with sterile PBS-, and incubate the cells for $2 \mathrm{~min}$ with $3 \mathrm{~mL}$ of PBS $-/ 0.02 \% \mathrm{EDTA}$ at room temperature. As soon as the cells begin to detach, remove the PBS $-/ 0.02 \%$ EDTA solution, and wash the plate with $6 \mathrm{~mL}$ of mTeSRl.

3. Pipet the cell solution to obtain small clusters, centrifuge cells at $200 \times g$ for $5 \mathrm{~min}$, and suspend in mTeSRl. 
4. Transfer the cell suspension to a suitable number of wells of a 6-well matrigel-coated culture plate. Culture the cells overnight (see Note 6).

5. Change the culture medium to RPMI 1640 medium supplemented with $2 \%$ B27 (without insulin), $100 \mathrm{ng} / \mathrm{mL}$ Activin A, $10 \mathrm{ng} / \mathrm{mL} \mathrm{BMP} 4$, and $20 \mathrm{ng} / \mathrm{mL} \mathrm{FGF} 2$, and culture for 2 days with daily medium changes.

6. Substitute the culture medium again to RPMI 1640 with $2 \%$ B27 (without insulin), $100 \mathrm{ng} / \mathrm{mL}$ Activin A for 3 days with daily medium changes.

7. Culture the definitive endoderm cells with RPMI 1640 containing $2 \% \mathrm{~B} 27$ (with insulin), $20 \mathrm{ng} / \mathrm{mL} \mathrm{BMP} 4$, and $10 \mathrm{ng} / \mathrm{mL}$ FGF-2 for 5 days with daily medium changes.

8. Culture the hepatic progenitor cells with RPMI 1640 containing $2 \% \mathrm{~B} 27$ (with insulin) and $20 \mathrm{ng} / \mathrm{mL}$ HGF for 5 days with daily medium changes.

By day 16 of the differentiation protocol, the cells should display a morphology that resembles hepatoblasts, with $80-90 \%$ of the cells expressing AFP and, quite commonly, some lipid droplets within the cytoplasm of the cells.

3.6 Scaffold Preparation and Sterilization for Bioreactor

3.7 Bioreactor Assembly and Recellularization
1. After liver decellularization, the left and central lobes are removed, to decrease the size of the scaffold and, consequently, the total cells needed to recellularize it.

2. A 4-0 silk suture is passed through the vascular structure of the left lobe to ligate it. The vessels are then cut, and the lobe is removed. This same procedure is performed to remove the central lobe.

3. The diaphragm should be trimmed as much as possible.

4. The remnant right lobe scaffold is then put in a $150 \mathrm{~cm}$ culture dish with $20 \mathrm{~mL}$ of distilled water.

5. The scaffold is then sterilized with X-ray at $160 \mathrm{kV}, 6.3 \mathrm{~mA}$, for $99.9 \mathrm{~min}$ (see Note 7).

For liver recellularization, it is necessary to assemble the setup described in Fig. 3. The process consists of three phases: the first is the priming, the second is cell seeding, and the third is the maintenance of the recellularized scaffold (for 7 days).

1. All the components used for the bioreactor setup are sterilized (see Note 8).

2. All the components are assembled in a culture biosafety cabinet, using sterile gloves, sterile lab coat, and a mask, to reduce contamination. 
Iris Pla-Palacín et al.

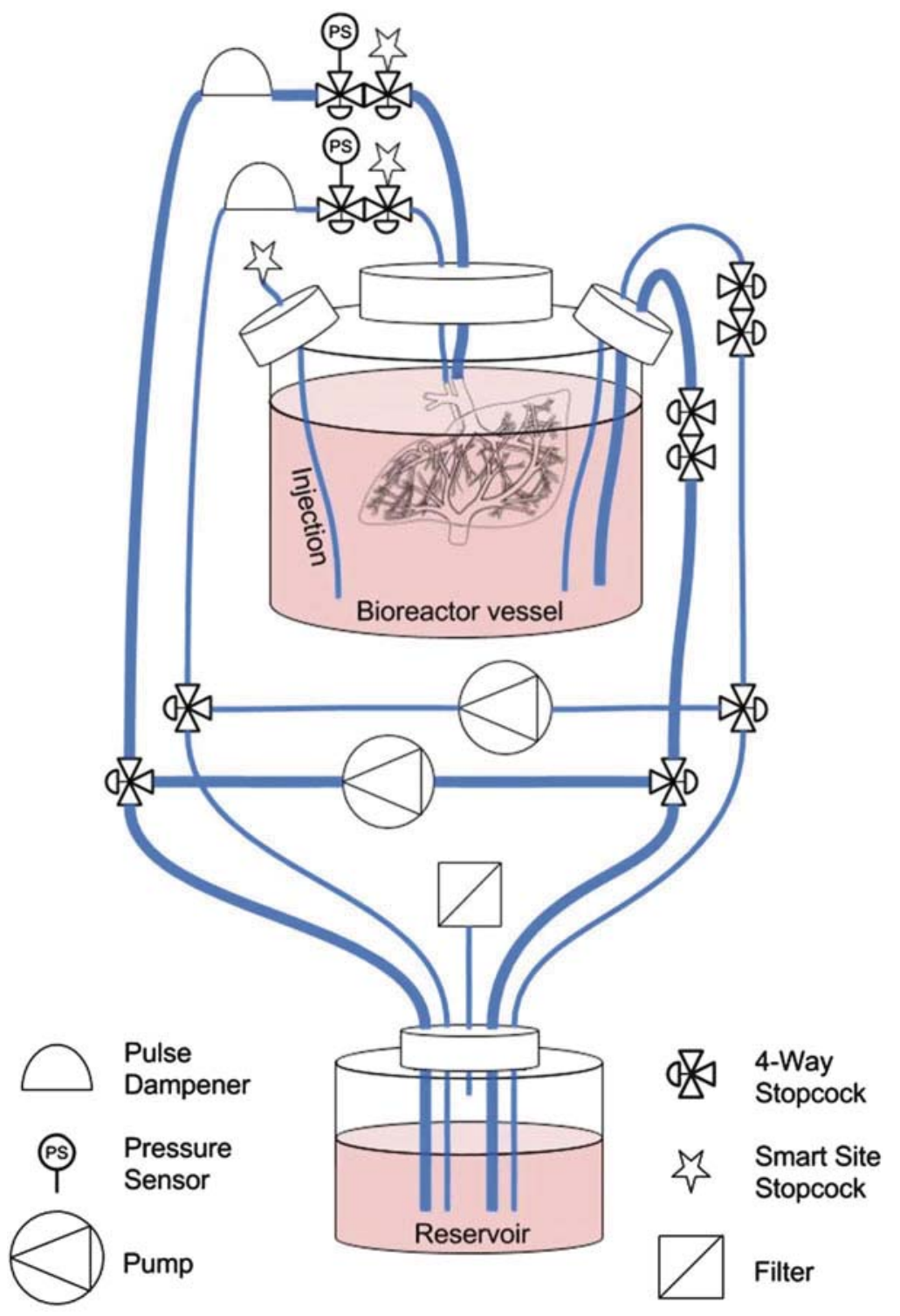

Fig. 3 Schematic diagram of the bioreactor system. Cells are injected through the injection tube to the media within the spinner flask. Then, they are perfused by the peristaltic pumps all across the circuit to the liver, entering the organ through the portal vein and the hepatic artery

3. The tubing is connected to the four-way stopcocks and smart site stopcocks using male and female Luer locks. The pulse dampeners are linked to the tubing.

4. Once the tubing is connected to the bioreactor vessel and the reservoir and the circuit is closed, it is primed with culture medium using a $20 \mathrm{~mL}$ Luer-lock syringe, to remove the air from the system. 
3.8 pUVEC

Preparation for

Bioreactor Seeding

3.9 pMSC

Preparation for

Bioreactor Seeding

3.10 iPS-Derived

Hepatoblast

Preparation for

Bioreactor Seeding
Liver Bioengineering Using Decellularized Whole-Liver Scaffolds

5. The liver is attached to the tubing through the portal fitting and the hepatic cannula and is suspended in the culture medium within the spinner flask.

6. The bioreactor system is transferred to an incubator at $37^{\circ} \mathrm{C} /$ $5 \% \mathrm{CO}_{2}$ and attached to the peristaltic pumps.

7. The liver is then perfused at low-pressure conditions overnight before the cell seeding: $25 \mathrm{mmHg}$ in both lines.

1. Aspirate the EGM-2 media from the $150 \mathrm{~cm}$ culture dish, and wash with $10 \mathrm{~mL}$ of PBS without calcium and magnesium.

2. Add $7 \mathrm{~mL}$ of trypsin/EDTA and incubate for $4 \mathrm{~min}$ at $37^{\circ} \mathrm{C}$.

3 . Collect the trypsin with the detached cells, and transfer to a $50 \mathrm{~mL}$ conical tube.

4. Rinse the dish with $7 \mathrm{~mL}$ of DMEM $+10 \% \mathrm{FBS}+1 \% \mathrm{P} / \mathrm{S}$ for any leftover cells, and transfer to the $50 \mathrm{~mL}$ conical tube.

5. Centrifuge at $300 \times g$ for $5 \mathrm{~min}$. Aspirate supernatant and reconstitute the pellet with $10 \mathrm{~mL}$ of bioreactor media.

6. Cell counting.

7. Keep the cells on ice until ready to be injected in the bioreactor system.

1. Aspirate the DMEM/F12 media from the $150 \mathrm{~cm}$ culture dish, and wash with $10 \mathrm{~mL}$ of PBS without calcium and magnesium.

2. Add $7 \mathrm{~mL}$ of trypsin/EDTA and incubate for $4 \mathrm{~min}$ at $37^{\circ} \mathrm{C}$.

3. Collect the trypsin with the detached cells and transfer to a $50 \mathrm{~mL}$ conical tube.

4. Rinse the dish with $7 \mathrm{~mL}$ of DMEM with $10 \% \mathrm{FBS}$ and $1 \% \mathrm{P} / \mathrm{S}$ for any leftover cells, and transfer to the $50 \mathrm{~mL}$ conical tube.

5. Centrifuge at $300 \times g$ for $5 \mathrm{~min}$. Aspirate supernatant and reconstitute the pellet with $10 \mathrm{~mL}$ of bioreactor media.

6. Cell counting.

7. Keep the cells on ice until ready to be injected in the bioreactor system.

1. Aspirate the media.

2. Add $3 \mathrm{~mL}$ of TrypLE for $10 \mathrm{~min}$ at room temperature.

3. Collect the TrypLE with the detached cells, and transfer to a $50 \mathrm{~mL}$ of conical tube.

4. Inactivate with DMEM/F12 with $10 \% \mathrm{FBS}$ and $1 \% \mathrm{P} / \mathrm{S}$.

5. Centrifuge $200 \times g$ at room temperature, and reconstitute the pellet with $10 \mathrm{~mL}$ of bioreactor media.

6. Cell counting. 
Iris Pla-Palacín et al.

7. Keep the cells on ice until ready to be injected in the bioreactor system.

Once all cell types are detached and counted, they should be centrifuged again. Pellets should be mixed with $10 \mathrm{~mL}$ of bioreactor media for scaffold seeding and supernatants discarded.

3.11 Scaffold Seeding
1. An ammount of approximately 60 million hepatoblasts, 45 million pUVECs and 12 million pMSCs (ratio 10:7:2) are co-seeded through the portal vein and the hepatic artery of the piglet liver scaffold by perfusion at $60 \mathrm{mmHg}$ in the hepatic artery and $25 \mathrm{mmHg}$ in the portal vein.

2. All types of cells used are put together in $10 \mathrm{~mL}$ of bioreactor media (see Note 9) and injected into the bioreactor through the injection tube. Peristaltic pumps will recirculate the cells to the portal vein and hepatic artery.

3. Cells are seeded in two steps, separated by $4 \mathrm{~h}$.

4. After the second seeding, a maintenance pressure is set up for $\mathrm{l}$ week, to maintain a specific fluid flow inside the scaffold that allows the attached cells not to detach. A set point of $25 \mathrm{mmHg}$ will be used for both lines.

5. The scaffold is left in the bioreactor system for 1 week, changing media every 2 days.

6. After 1 week, the reseeded scaffold needs to be perfused with $4 \%$ paraformaldehyde for $15 \mathrm{~min}$, after which small pieces are taken for tissue processing.

\section{Notes}

1. Porcine infra-hepatic vena cava penetrates the liver right lobe. Some care is needed not to disrupt this hepatic lobe.

2. Depending on each liver, it might be necessary to prepare more or less detergent.

3. Colonies usually appear between 5 and 21 days after pUVECs isolation.

4. A four-in-one mono-cassette virus is recommended. $1 \mu \mathrm{g} / \mathrm{mL}$ of doxycycline should be added in every medium change to induce gene expression. The amount of virus added is calculated using a multiplicity of infection (MOI) of at least 20. To determine the desired amount of virus: virus volume $(\mu \mathrm{L})$ required $=[(\#$ pMSCs seeded for infection $) /$ virus titer $(\mathrm{U} / \mathrm{mL})] \times[($ desired MOI) $/ 1 \mathrm{~mL}] \times 1000 \mu \mathrm{L}$.

5. It is essential to start the differentiation protocol of iPCs cells into hepatoblast-like cells without minimal morphological evidence of differentiation. 
6. The cell density at the initiation of differentiation usually has a dramatic effect on differentiation efficiency, so the best density should be determined empirically.

7. It is desirable to use gamma irradiation for scaffold sterilization, but this procedure is not always available.

8. Tubing, spinner flask, and pulse dampener are sterilized by steam at $121{ }^{\circ} \mathrm{C}$. Some other components cannot be autoclaved: four-way stopcocks and smart connectors are sterilized with ethylene oxide; the pressure sensor is cleaned with a bactericide solution for $15 \mathrm{~min}$ and then washed with sterile PBS. The outer part is cleaned with ethanol.

9. Before loading the cell suspension into a Luer-lock syringe, it is vital to resuspend the cells carefully to reduce cell aggregation which could obstruct the scaffold vascular network.

In this chapter, we describe a seeding method in which cells are seeded with continuous perfusion. Some other authors have used multistep infusion systems, where cells are delivered directly inside the scaffold.

\section{Acknowledgments}

This work was supported by Instituto de Salud Carlos III, through a predoctoral fellowship i-PFIS IFI15/00158 (I. P-P), Gobierno de Aragón and Fondo Social Europeo through a predoctoral fellowship DGA C066/2014 (P. S-A), and by a predoctoral fellowship from Fundação para Ciência e Tecnologia (PD/BD/114057/ 2015), Portugal (S.M.). PMB was supported by an H2020-MSCAIF-2014 from the European Research Agency and by the PI15/ 00563 Research Project from Instituto de Salud Carlos III, Madrid, Spain.

\section{References}

1. Baptista PM et al (2011) The use of whole organ decellularization for the generation of a vascularized liver organoid. Hepatology 53:604-617

2. Soto-Gutierrez A et al (2011) A whole-organ regenerative medicine approach for liver replacement. Tissue Eng Part C Methods 17:677-686
3. Rajarajan K, Engels MC, Wu SM (2012) Reprogramming of mouse, rat, pig, and human fibroblasts into iPS cells. Curr Protoc Mol Biol. Chapter 23, Unit 2315

4. Mallanna SK, Duncan SA (2013) Differentiation of hepatocytes from pluripotent stem cells. Curr Protoc Stem Cell Biol 26, Unit lG 4 